\title{
Análise de sensibilidade dos métodos de estimativa da evapotranspiração de referência e razão de Bowen em cultura da cana-de-açúcar
}

\author{
Bruce K. N. Silva ${ }^{1}$, Vicente de P. R. da Silva ${ }^{1}$, \\ Pedro V. de Azevedo ${ }^{1} \&$ Carlos H. de A. Farias ${ }^{2}$
}

\begin{abstract}
RESU M 0
A técnica de análise de sensibilidade de modelos foi aplicada nos dados obtidos em experimento de campo conduzido no ano de 2009, na Destilaria M iriri, localizada no município de Capim, PB, e aos dados de evapotranspiração de referência $\left(E_{0}\right)_{0}$ ), obtidos pela equação de Penman-M onteith (FAO/56), e da evapotranspiração da cultura da cana-de-açúcar (ET), obtidos pelo balanço de energia com base na razão de Bowen. As análises de sensibilidade e de erros foram aplicadas ao método da razão de Bowen para os intervalos de medição de 15, 30,60 e 120 minutos enquanto o método de Penman-Monteith foi aplicado para a escala diária durante 0 ano de 2009. O s resultados evidenciam que o saldo de radiação é a variável mais sensitiva no cálculo do balanço de energia, enquanto o fluxo de calor no solo oferece a menor contribuição; já os erros relativos de todas as variáveis envolvidas do cálculo balanço de energia baseado na razão de Bowen aumentaram significativamente com o aumento do intervalo de amostragem. A variável mais sensível na determinação da ET saldo de radiação seguida da umidade relativa, velocidade do vento a $2 \mathrm{~m}$ de altura e a temperatura média do ar.
\end{abstract}

Palavras-chave: balanço de energia, método de Penman-Monteith, análise de erros

\section{Sensitivity analysis of methods for estimating reference evapotranspiration and sugarcane evapotranspiration}

\begin{abstract}
A B ST RA C T
The sensitivity analysis technique of models was applied to the data obtained from field experiment carried out during 2009 at Distillery Miriri, Capim, PB. This technique was applied to the reference evapotranspiration (ET) by Penman-M onteith (FAO/56) and the sugarcane evapotranspiration (ET) by energy balance equation based on Bowen ratio. The sensitivity analysis and errors were applied to the Bowen ratio method in measurement intervals of 15, 30, 60 and 120 min; however Penman-M onteith approach was applied on daily basis throughout the 2009 year. Results showed that the net radiation is the most sensitive variable in the energy equation balance and soil heat flux offers the low est contribution. On the other hand, the relative errors for all variables in energy balance equation increase significantly with increase in sampling interval. The most sensitive variable in reference evapotranspiration by PenmamMonteith method is net radiation, followed by relative humidity, wind speed at $2 \mathrm{~m}$ and air temperature.
\end{abstract}

Key words: energy balance, Penman-M onteith method, error analysis 


\section{INTRODUÇÃO}

Comumente, o método do balanço de energia é utilizado para se estimar a evapotranspiração de superfícies vegetadas e se baseia nas análises do balanço energético dos ganhos e perdas de energia térmica radiativa, condutiva e convectiva por uma superfície evaporante. O método da razão de Bowen tem sido intensamente utilizado por diversos pesquisadores, com a finalidade de resolver a equação do balanço de energia sobre culturas e, assim, determinar a evapotranspiração (Azevedo et al., 2003; Silva et al., 2007; Borges et al., 2008; Souza et al., 2008). A estimativa adequada da evapotranspiração da cultura $\left(\mathrm{ET}_{\mathrm{c}}\right)$ consiste no principal parâmetro a ser considerado no dimensionamento e no manejo de sistemas de irrigação, uma vez que ela totaliza a quantidade de água utilizada nos processos de evaporação e transpiração pela cultura durante determinado período (Hurst et al., 2004; Silva et al., 2009). Em muitas aplicações práticas a ET é obtida através do valor da evapotran spiração de referência ( $\left.\mathrm{ET}_{\mathrm{o}}\right)$ corrigida pelo coeficiente da cultura $\left(\mathrm{K}_{\mathrm{c}}\right)$ que é dependente do tipo de cultura e do seu estágio de desenvolvimento (Sousa et al., 2010).

As variáveis climáticas são submetidas a diferentes tipos de erros em modelos agrometeorológicos. A primeira fonte de erro é devida às propriedades dos sensores, calibração e manuseio dos instrumentos, a segunda é devida à estimativa de certa variável climática em função de outra e, finalmente, a terceira fonte de erros está relacionada com a frequência temporal de coleta dos dados climáticos (Hupet \& Vanclooster, 2001). A técnica de análise de sensibilidade tem sido aplicada em várias áreas do conhecimento, principalmente em ciências ambientais, onde os modelos computacionais são utilizados. Rana \& Katerji (1998) analisaram a sensibilidade da equação de PenmanMonteith aos fatores climáticos (energia disponível e déficit de pressão de vapor) e da cultura (resistência estomática e aerodinâmica) em um clima semiárido, no sul da Itália. Os resultados desse estudo esclareceram que a energia disponível e a resistência aerodinâmica são as maiores fontes de erro na

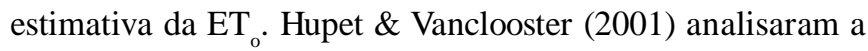
sensibilidade da equação de Penman-Monteith aos dados climáticos e calcularam os efeitos da variação da frequência temporal das variáveis sobre a estimativa da ET diária. Por outro lado, Xu et al. (2006) utilizaram a mesma técnica para analisar os impactos das variáveis meteorológicas no cálculo da evapotranspiração de referência pelo método de PenmanMonteith na bacia hidrográfica de Changjian e obtiveram que a variável mais sensível à equação de Penman-Monteith na área de estudo foi a umidade do ar seguida pela radiação de ondas curtas e temperatura do ar; já a velocidade do vento apresentou os menores valores de coeficiente de sensibilidade, explicados pela sua grande variabilidade espacial na região durante o período de estudo. Ainda com base no argumento de que a análise de sensibilidade é uma ferramenta importante para o entendimento das variações das variáveis no cálculo da ET, Gong et al. (2006) utilizaram a técnica de análise de sensibilidade para determinar uma combinação melhor entre a evapotranspiração e o escoamento superficial, avaliando os parâmetros mais sensíveis, comparadoa à ET determinada pelos métodos de Hargreaves e de Penmnm-Montheit.
Gong et al. (2006) realizaram um estudo com a equação de Penman-Monteith utilizando dados de 150 estações meteorológicas no período de 1960 a 2000. Esse estudo esclareceu que, em geral, a umidade relativa do ar é a principal fonte de erros para o modelo seguida da radiação de ondas curtas, temperatura do ar e velocidade do vento. Liqiao et al. (2008) também analisaram os coeficientes de sensibilidade das variáveis de entrada do modelo de Penman-Monteih durante o período de 1961 a 2005 para uma bacia hidrográfica no nordeste da China e concluíram que a umidade relativa do ar é o principal fator controlador da evapotranspiração dentre as variáveis climáticas exigidas por este modelo.

Todos os estudos aqui mencionados se referem ao modelo de Penman-Monteith; entretanto, a análise de sensibilidade de modelos de estimativa de evapotranspiração de culturas se reveste de grande importância na área agronômica, em especial no manejo da irrigação. Apesar da importância do estudo, a análise de sensibilidade aplicada à equação do balanço de energia é ainda incipiente. Neste sentido, este trabalho tem o objetivo de elaborar a análise de sensibilidade e de erros na estimativa da evapotranspiração de referência pelo método de Penman-Monteith e na razão de Bowen na cultura da cana-deaçúcar.

\section{Material e MÉTODOS}

A pesquisa foi realizada no ano de 2009 no município de Capim, localizado na microrregião do Litoral Norte do Estado da Paraíba. O experimento de campo foi conduzido na Miriri Alimentos e Bioenergia S/A, situada geograficamente entre as latitudes $6^{\circ} 56^{\prime} \mathrm{S}$ e $35^{\circ} 07^{\prime} \mathrm{W}$, dentro de uma região propícia ao cultivo da cultura da cana-de-açúcar, com altitude em torno de $54 \mathrm{~m}$ e temperatura média de $28^{\circ} \mathrm{C}$. A precipitação média anual é em torno de $1.600 \mathrm{~mm}$, com seis meses secos; o clima é quente e úmido, com chuvas de outono a inverno (Silva, 2004). O solo da área experimental é classificado como Argissolo vermelhoamarelo e possui textura média, franco-argilo-arenosa, com capacidade de armazenar, em média, $61 \mathrm{~mm}$ de água. A pesquisa foi desenvolvida com a variedade RB 92579, no primeiro ciclo de produção (cana-planta), numa parcela de sequeiro, durante dias representativos de cada estádio fenológico da cultura.

$\mathrm{Na}$ parcela experimental foi instalada uma torre micrometeorológica com os seguintes instrumentos sobre a cultura da cana-de-açúcar: dois anemômetros de conchas (modelo 03001, marca Young), em dois níveis, um localizado a $0,50 \mathrm{~m}$ e o outro a $1,95 \mathrm{~m}$, ambos acima da copa da cultura, para medição da velocidade do vento; dois piranômetros (modelo CM3, marca Kipp \& Zonen), a 1,95 m, para medições da radiação solar incidente e refletida acima do dossel vegetativo; um saldo radiômetro (modelo NR lite, marca Kipp \& Zonen), posicionado sobre a copa das plantas para medição do saldo de radiação $\left(\mathrm{R}_{\mathrm{n}}\right)$; dois psicrômetros não aspirados de cobre-constantan devidamente calibrados, para medição das temperaturas dos bulbos seco e úmido, situados a 0,6 e 2,9 m acima do dossel vegetativo e um sensor para medição da temperatura e umidade relativa do ar (modelo HMP45C, marca Campbell Scientific, Inc.). 
Também foram instalados dois fluxímetros (modelo HFT3, marca Campbell Scientific, Inc.) para medições do fluxo de calor no solo $(\mathrm{G})$ posicionados a $2 \mathrm{~cm}$ de profundidade. $\mathrm{O}$ método da razão de Bowen foi utilizado para determinar os componentes do balanço de energia sobre o canavial em duas datas do período seco e duas no período chuvoso. As datas selecionadas para as análises de erros e de sensibilidade dos componentes do balanço de energia foram 19 de março, 09 de maio, 30 de agosto e 01 de setembro de 2009 . O perfil de temperatura do ar foi medido no interior do dossel da planta selecionada, por meio de termopares de cobre-constantan instalados a 1,0;2,0 e 3,0 metros de altura. Todos os sensores foram conectados a um sistema automático de aquisição e armazenamento de dados (Datalogger CR 23X, Campbell Scientific, Inc.), programado por meio de linguagem específica com varredura dos sensores a cada cinco segundos e médias armazenadas a cada 15 minutos.

A evapotranspiração de referência $\left(\mathrm{ET}_{\mathrm{o}}\right)$ foi obtida pelo método da FAO Penman-Monteith, considerando-se a resistência estomática de $70 \mathrm{sm}^{-1}$ e a altura da cultura hipotética fixada em $0,12 \mathrm{~m}$ e albedo de $23 \%$ pela equação (Allen et al., 1998):

$$
\text { ETo }=\frac{0,408 \Delta\left(\mathrm{R}_{\mathrm{n}}-\mathrm{G}\right)+\gamma\left(\frac{900 \mathrm{U}_{2}}{\mathrm{~T}+273}\right)\left(\mathrm{e}_{\mathrm{s}}-\mathrm{e}_{\mathrm{a}}\right)}{\Delta+\gamma\left(1+0,34 \mathrm{U}_{2}\right)}
$$

em que:

$\mathrm{ET}_{\mathrm{o}}$ é expresso em $\mathrm{mm}$ dia $^{-1}$; já $\mathrm{R}_{\mathrm{n}}$ (saldo de radiação) e $\mathrm{G}$ (densidade do fluxo de calor no solo) são expressos em $\mathrm{MJ} \mathrm{m}^{-2} \mathrm{~d}^{-1}$

$\Delta$ - declinação da curva de saturação do vapor da água, $\mathrm{kPa}{ }^{\circ} \mathrm{C}^{-1}$

$\mathrm{U}_{2}$ - velocidade do vento (média diária) a $2 \mathrm{~m}$ acima da superfície do solo, $\mathrm{m} \mathrm{s}^{-1}$

$\mathrm{T}$ - temperatura do ar, ${ }^{\circ} \mathrm{C}$

$\mathrm{e}_{\mathrm{s}}$ - pressão de saturação do vapor, $\mathrm{kPa}$

$\mathrm{e}_{\mathrm{a}}$ - pressão real do vapor, $\mathrm{kPa}$

$\gamma$ - fator psicométrico, $\mathrm{MJ} \mathrm{kg}^{-1}$

Os dados da estação agrometeorológica do Instituto Nacional de Pesquisas Espaciais (INPE) instalada a cerca de $1 \mathrm{~km}$ do local do experimento, foram coletados no site www.inpe.br para a determinação da evapotranspiração de referência.

O balanço de energia é baseado no princípio de conservação de energia, dado pela seguinte expressão:

$$
\mathrm{R}_{\mathrm{n}}=\mathrm{LE}+\mathrm{H}+\mathrm{G}+\mathrm{S}+\mathrm{P}
$$

em que:

$\mathrm{R}_{\mathrm{n}}$ - saldo de radiação (energia disponível)

$\mathrm{LEe} \mathrm{H}$-fluxos verticais de calor latente e sensível, respectivamente

$\mathrm{G}$ - fluxo de calor no solo e S é a energia armazenada no dossel vegetativo

$\mathrm{P}$ - fotossíntese.

O termo $\mathrm{P}$ não foi considerado porque ele representa menos de $2 \%$ do saldo de radiação. Todos os termos da Eq. (2) apresentam as unidades em $\mathrm{W} \mathrm{m}^{-2}$. Considerou-se, também, que as densidades de fluxo vertical que chegam à camada vegetativa são positivas, enquanto as que saem são negativas.

O fluxo de calor latente (LE) foi obtido substituindo-se a razão de Bowen na Eq. (2), portanto:

$$
\mathrm{LE}=\frac{\mathrm{R}_{\mathrm{n}}+\mathrm{G}+\mathrm{S}}{1+\beta}
$$

A equação acima permite a estimativa de LE com base em medições do fluxo de calor sensível. O termo $\beta$, que é o termo da razão de Bowen, corresponde à fração de energia entre os fluxos de calor sensível e calor latente, dado por:

$$
\beta=\frac{H}{L E}
$$

A Eq. (4) pode ser escrita na forma:

$$
\beta=\frac{\mathrm{Pc}_{\mathrm{P}}}{\mathrm{L} \in}\left(\frac{\mathrm{K}_{\mathrm{h}}}{\mathrm{K}_{\mathrm{w}}}\right) \frac{\partial \mathrm{T} / \partial \mathrm{Z}}{\partial \mathrm{e} / \partial \mathrm{Z}}=\gamma \frac{\mathrm{K}_{\mathrm{h}}}{\mathrm{K}_{\mathrm{w}}} \frac{\Delta \mathrm{T}}{\Delta \mathrm{e}}
$$

em que:

$\gamma \quad$ - fator psicrométrico, $\mathrm{kPa}^{\circ} \mathrm{C}^{-1}$

$\Delta \mathrm{T}$ - gradiente da temperatura do ar, ${ }^{\circ} \mathrm{C}$

$\Delta \mathrm{e}$ - gradiente da pressão parcial do vapor d'água

$\mathrm{K}_{\mathrm{h}}$ e $\mathrm{K}_{\mathrm{w}}$ - coeficientes de difusão turbulenta de calor sensível e de vapor d'água, respectivamente. Na ausência de advecção de calor sensível, regional ou local, e em condição de instabilidade atmosférica, $\mathrm{K}_{\mathrm{h}}=\mathrm{K}_{\mathrm{w}}$ Calculou-se a pressão parcial de vapor d'água em dois níveis acima da copa das plantas pela equação de Ferrel, cujos procedimentos são dados em Borges et al. (2008).

O calor armazenado pelo dossel vegetativo foi calculado com base em medidas do gradiente de temperatura do ar, em três níveis no interior do dossel da cultura. No cálculo dessa variável foi utilizada a seguinte equação (Borges et al., 2008):

$S=\frac{\left[\sum \rho_{\mathrm{a}} \mathrm{C}_{\mathrm{p}} \Delta \mathrm{h}\left(\frac{\mathrm{T}_{\mathrm{i}}+\mathrm{T}_{\mathrm{i}+1}}{2}\right)_{\mathrm{j}+1}-\sum \rho_{\mathrm{a}} \mathrm{C}_{\mathrm{p}} \Delta \mathrm{h}\left(\frac{\mathrm{T}_{\mathrm{i}}+\mathrm{T}_{\mathrm{i}+1}}{2}\right)\right]}{900}$

em que:

$\rho_{\mathrm{ar}}$ - densidade do ar, $1,3 \mathrm{~kg} \mathrm{~m}^{-3}$

$\mathrm{C}_{\mathrm{p}}$ - calor específico do ar a pressão constante, $1005 \mathrm{~J} \mathrm{~kg}^{-1} \mathrm{C}^{-1}$

$\Delta \mathrm{p}$ - distância vertical entre os sensores de medidas, $1 \mathrm{~m}$

$\mathrm{Ti}$ - temperatura do ar numa altura "i" e “j”, que representa cada instante em que se calculou a temperatura média da camada de ar

Assim, foi obtida a energia térmica do ar nesse instante, em cada uma das camadas do dossel vegetativo. O calor 
armazenado no dossel do canavial foi obtido pela diferença entre as somas das energias térmica de cada uma das camadas, estimadas entre cada intervalo de $15 \mathrm{~min}$, dividindo-se o resultado pelo tempo em segundos, ou seja, $900 \mathrm{~s}$.

O erro relativo devido à variação temporal dos intervalos de medição foi calculado conforme Hupet \& Vanclooster (2001):

$$
\varepsilon_{\text {rel }}=\frac{\left.<\operatorname{var}>_{\mathrm{i}}-<\operatorname{var}\right\rangle_{\text {med }}}{<\operatorname{var}>_{\text {med }}} 100 \%
$$

e o erro absoluto por

$$
\varepsilon_{\text {abs }}=<\text { var }>_{i}-<\text { var }>_{\text {med }}
$$

em que:

<var $\rangle_{i}$ - valor da variável do intervalo de medição i

<var ${ }_{\text {med }}$ - média da variável usando-se o intervalo mínimo de medição

Nos modelos multivariados, como os de Penman-Monteith e balanço de energia, dentre outros, as variáveis têm dimensões e variam em intervalos diferentes, o que torna difícil a comparação da sensibilidade das variáveis através de derivadas parciais. Assim, tais derivadas devem ser transformadas para a forma não-dimensional (Beven, 1979) para se avaliar os efeitos dos erros das variáveis sobre o modelo. O coeficiente de sensibilidade relativa não dimensional de cada variável pode ser obtido pela seguinte equação (McCuen, 1974):

$$
\mathrm{S}_{\mathrm{i}}=\frac{\partial \mathrm{ET}}{\partial \mathrm{v}_{1}} \frac{\mathrm{v}_{\mathrm{i}}}{\mathrm{ET}}
$$

Os coeficientes de sensibilidade obtidos pela Eq. (9) representam a fração de variação de $v_{i}$ transmitida à variação de ET e LE. As derivadas parciais necessárias para a determinação dos coeficientes de sensibilidade foram calculadas analiticamente através de software matemático. Os coeficientes de sensibilidade foram obtidos para os intervalos de tempo descritos nesta seção do trabalho. Os valores positivo/negativo do coeficiente de sensibilidade de uma variável indicam que a função aumenta/decresce quando o valor da variável aumenta. Na forma gráfica o coeficiente de sensibilidade representa a inclinação da curva de sensibilidade já que o coeficiente é suficientemente preciso dentro de certo intervalo linear (Gong et al., 2006).

\section{RESULTADOS E DISCUSSÃO}

A evolução diária da evapotranspiração de referência (ET), no período de 01/09/2008 a 30/09/2009, é apresentada na Figura 1. Os menores valores de ET variaram entre $1,7 \mathrm{~mm} \mathrm{~d}^{-1} \mathrm{em}_{\mathrm{o}}$ abril a $1,10 \mathrm{~mm} \mathrm{~d}^{-1}$ em agosto de 2009 , em virtude da pouca disponibilidade de energia no período chuvoso. No período seco observou-se, na região com pouca umidade no solo, um aumento na ET.

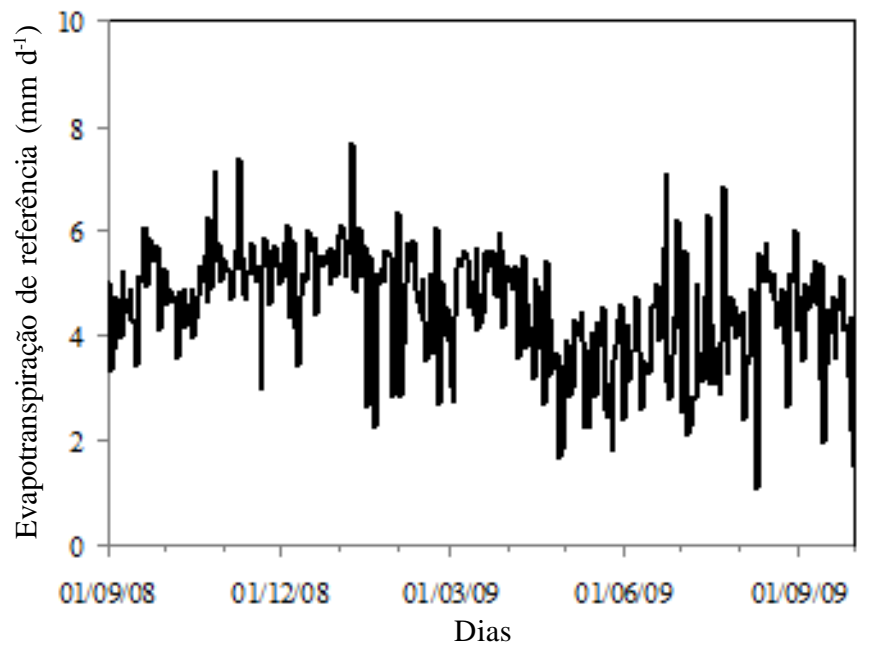

Figura 1. Evapotranspiração de referência diária determinada pelo método Penman-M onteith, para o período de 1/9/2008 a 30/09/2009

Os maiores valores da evapotranspiração foram encontrados nos períodos de setembro de 2008, fevereiro, julho e setembro de 2009, com valores superiores a 7,0 $\mathrm{mm} \mathrm{d}^{-1}$, período que corresponde à época de alta demanda atmosférica e também de maior disponibilidade de água no solo através da precipitação ou irrigação.

Os valores dos coeficientes de sensibilidade das variáveis envolvidas no cálculo da evapotranspiração de referência variaram consideravelmente durante o período de estudo. $\mathrm{O}$ curso anual dos valores dos coeficientes de sensibilidade do saldo de radiação $\left(\mathrm{S}_{\mathrm{Rn}}\right)$, temperatura do $\operatorname{ar}\left(\mathrm{S}_{\mathrm{T}}\right)$, umidade relativa $\left(S_{E}\right)$ e velocidade do vento $\left(S_{U}\right)$ estão representados na Figura 2. Observa-se que os maiores valores do coeficiente de sensibilidade são para o saldo de radiação, que variaram de

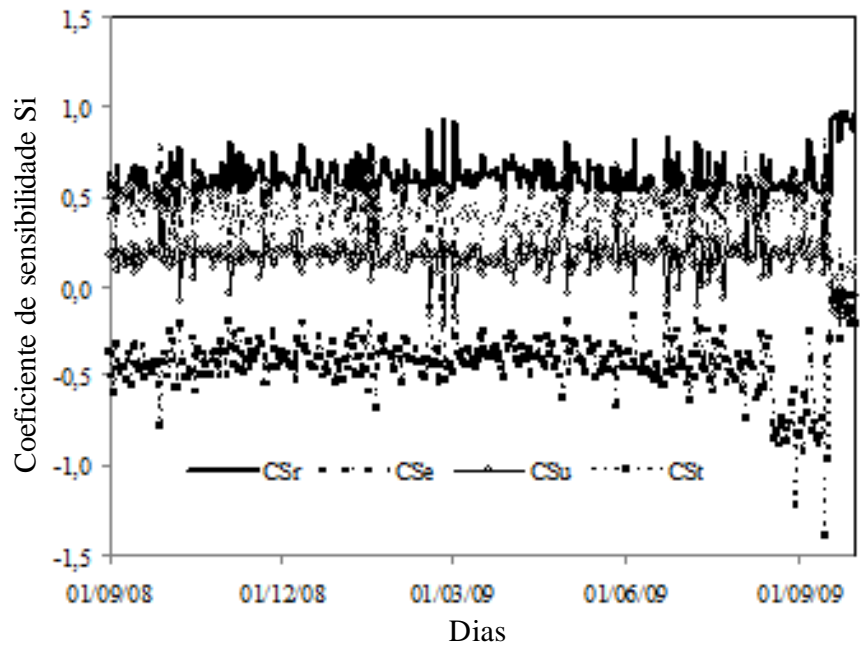

Figura 2. Variabilidade dos coeficientes de sensibilidade das variáveis envolvidas no cál culo da evapotranspiração de referência diária pelo método parametrizado de Pemman-M onteith. CSr é o coeficiente de sensibilidade do saldo de radiação, CSe é da umidade do ar, CSu da velocidade do vento e CSt o coeficiente de sensibilidade da temperatura média do ar 
0,19 a 0,97 . Por outro lado, os valores de $S_{E}$ variam de 0,03 a 0,81 e de $S_{U}$ entre -0,22 e 0,6, no final de setembro de 2009.

O coeficiente $S_{T}$ apresentou variação negativa, ou seja, ele foi o que menos contribuiu para o aumento da sensibilidade na estimativa de $\mathrm{ET}_{\mathrm{o}}$ no período estudado, com valores entre -1,4 e $-0,04$ ao longo de todo o período. Esses resultados se aproximam daqueles obtidos por Hupet \& Vanclooster (2001), ao evidenciarem que a radiação solar é a variável atmosférica que mais influencia o cálculo da ET . Por outro lado, Xu et al. (2006) obtiveram que a variável mais sensível a essa equação na sua área de estudo foi a umidade relativa do ar seguida de radiação de ondas curtas e temperatura do ar; já a velocidade do vento apresentou os menores valores de coeficientes de sensibilidade, que foram explicados pela sua grande variabilidade espacial na região.

Os fluxos de energia foram calculados para o período diurno, quando o saldo de radiação foi positivo, procedimento também adotado por Azevedo et al. (2003), Borges et al. (2008). Na Figura 3 se apresenta o balanço de energia para dois dias representativos do período experimental.

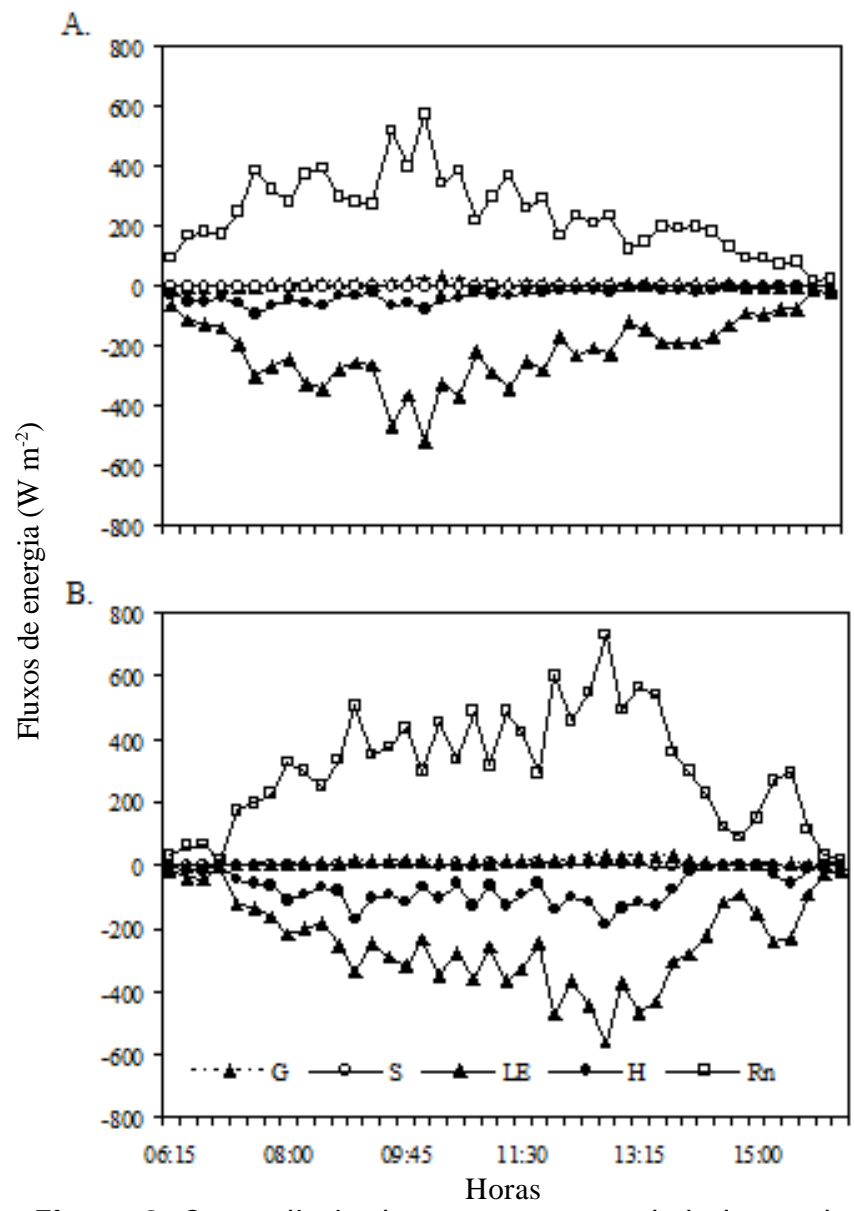

Figura 3. Curso diário dos componentes do balanço de energia sobre a cultura da cana-de-açúcar em 01 de setembro 2009 (A) e em 19 de março de 2009 (B)

Verificou-se, no dia 01 de setembro de 2009, intensa variabilidade do saldo de radiação em face da alta nebulosidade registrada nesse dia (Figura 3A). Os pontos máximos das densidades de fluxo de calor latente (LE) e de $\mathrm{R}_{\mathrm{n}}$ ocorrem próximo às $11 \mathrm{~h}$, com valores de 519 e $576 \mathrm{~W} \mathrm{~m}^{-2}$, respectivamente. Os fluxos de calor sensível $(\mathrm{H})$ apresentaram, até o meio dia, uma variação bem menor, atingindo valor médio de $-23,5 \mathrm{~W} \mathrm{~m}^{-2}$; já os fluxos de calor no solo $(\mathrm{G})$ e de calor armazenado no dossel vegetativo (S), atingiram valores médios de 0,61 e $6,0 \mathrm{~W} \mathrm{~m}^{-2}$, respectivamente. Por outro lado, os fluxos de $\mathrm{R}_{\mathrm{n}}$ apresentaram valores máximos por volta das $15 \mathrm{~h} 30 \mathrm{~min}$, no dia 19 de maio (Figura 3B). Borges et al. (2008), que também aplicaram a técnica do balanço de energia à cultura da mangueira, encontraram valores médios de $\mathrm{R}_{\mathrm{n}}$ e LE de 400 e 300 $\mathrm{W} \mathrm{\textrm {m } ^ { - 2 }}$, respectivamente, superiores àqueles encontrados nesta pesquisa $\left(\mathrm{R}_{\mathrm{n}}=307 \mathrm{~W} \mathrm{~m}^{-2} \mathrm{e} \mathrm{LE}=243 \mathrm{~W} \mathrm{~m}^{-2}\right)$.

Os coeficientes de sensibilidade do fluxo de calor no solo $(\mathrm{Sg})$, do saldo de radiação $(\mathrm{Sr})$, da temperatura do bulbo seco (St) e da temperatura do bulbo úmida (Se) no intervalo de medição de 15 min, são apresentados na Figura 4. O coeficiente de sensibilidade da temperatura do bulbo seco foi maior quando comparado com os das outras variáveis para os dias analisados, com máximo de 0,34 , no dia 19 de março e mínimo de -0,01, no dia 09 de maio. Para o dia 09 de maio (Figura 4 B), St variou de $-0,01$ a 0,34 , com média e desvio padrão de $0,22 \pm 0,08$ enquanto o coeficiente de sensibilidade do fluxo de calor no solo $(\mathrm{Sg})$ variou de -0,04 a 0, com média e desvio padrão de -0,02 e 0,01, respectivamente. Por outro lado, Se variou de -0,33 a 0,01, com média e desvio padrão de $-0,22 \pm 0,08$, respectivamente. Finalmente, $\mathrm{Sr}$ apresentou média e desvio padrão de $-0,98 \pm$ 0,02. Desta forma, os valores de St são consistentemente menores do que outras variáveis, em todos os dias analisados.

Os coeficientes de sensibilidade do saldo de radiação, temperatura do bulbo úmido e do fluxo de calor no solo, têm evoluções semelhantes e oscilam dentro da mesma ordem de magnitude. No dia 30 de agosto os coeficientes $\mathrm{Sg}$, Sr, St e Se apresentaram valores médios de $-0,01 ;-0,99 ; 0,1$ e $-0,1$, respectivamente (Figura $4 \mathrm{C}$ ). Similarmente, no dia 1 de setembro os valores de $\mathrm{Sg}, \mathrm{Sr}$, St e Se foram -0,02; -0,98; 0,11 e -0,11, respectivamente (Figura 4D). A eficiência da análise de sensibilidade foi constatada por Yeh \& Huang (2006), em estudo relacionado com aquífero para avaliar o melhor modelo visando determinar a distância do bombeamento até o poço, com base nessa técnica. No dia analisado no presente estudo, o maior valor foi para o coeficiente de sensibilidade da temperatura do bulbo seco, com o valor de 0,34 e o menor foi para o saldo de radiação, com valor de $-1,1$.

Observa-se ainda que a variabilidade dos coeficientes de todas variáveis é muito pequena ao longo do período diurno, no intervalo de medições de $15 \mathrm{~min}$. Os resultados obtidos por Gong et al. (2006) indicam que em algumas regiões da bacia de Changjian a variável que apresentou maior sensibilidade foi a temperatura média do ar seguida pela umidade relativa do ar. Assim, esses resultados são conflitantes com os obtidos neste trabalho, para o intervalo de tempo de medição de 15 min; este fato correu face, possivelmente, à variabilidade espacial dessa variável na Bacia de Changjian, China.

Quanto maior o valor absoluto do coeficiente de sensibilidade maior também será o efeito de determinada variável sobre o fluxo de calor latente (Liqiao et al., 2008). Assim, neste intervalo de medição e em termos de valor absoluto, os menores valores do coeficiente de sensibilidade foram para o fluxo de calor no solo e os maiores para o saldo de radiação. 


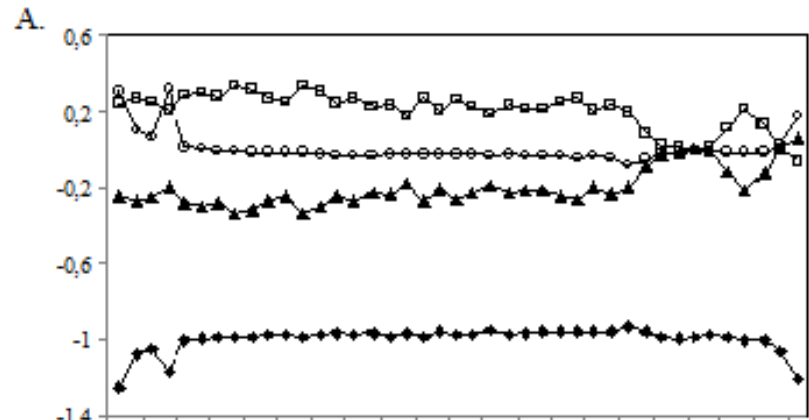

B.

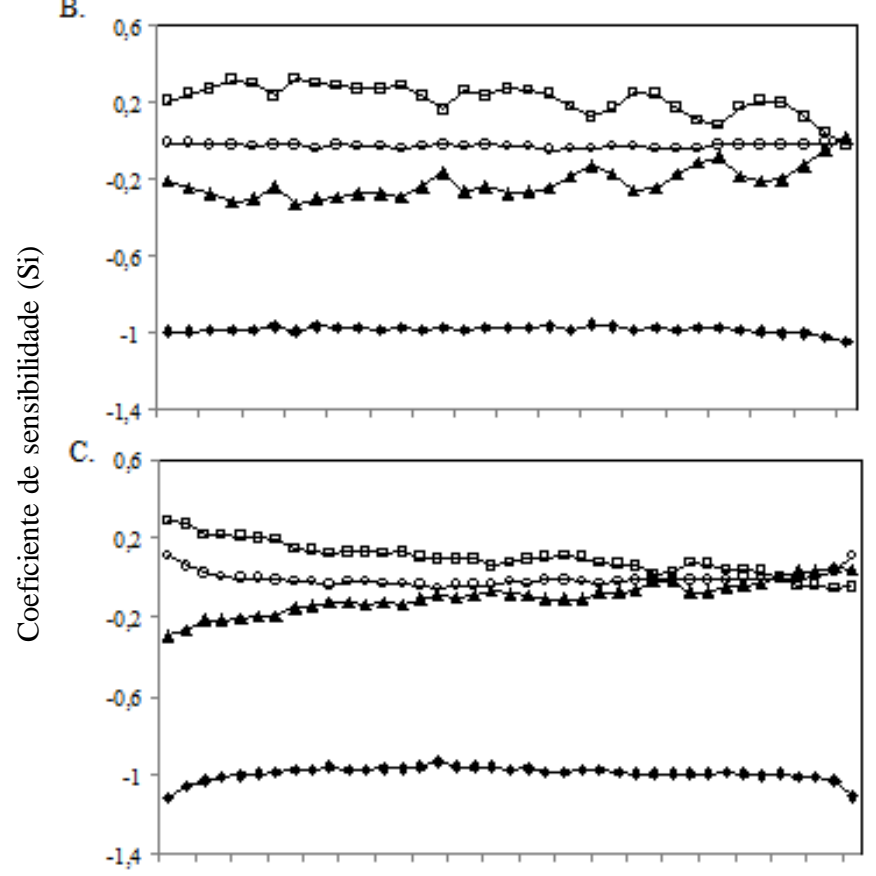

D.

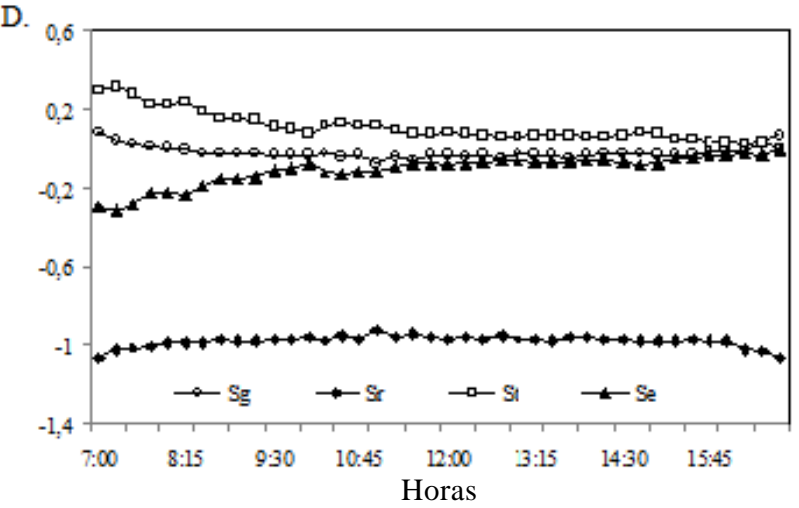

Figura 4. Curso temporal dos coeficientes de sensibilidade da evapotranspiração da cultura pelo método do balanço de energia baseado na razão de Bowen em intervalos de mediação de 15 minutos nos dia 19 de março (A), 9 de maio (B), 30 de agosto (C) e 1 de setembro (D) do ano de 2009

A justificativa para tal é que a variabilidade de $\mathrm{R}_{\mathrm{n}}$ é muito maior do que de $\mathrm{G}$ em face, sobremaneira, da variação da cobertura de nuvens. A temperatura do bulbo seco e a temperatura do bulbo úmido apresentaram graus de sensibilidade semelhantes no cálculo do fluxo de calor latente, variando entre $-0,21$ e 0,21 . A Tabela 1 descreve as médias dos valores absolutos dos coeficientes de sensibilidade das variáveis envolvidas no cálculo do balanço de energia, com base na razão de Bowen, dos quatro dias analisados no estudo, para cada intervalo de medição.

Tabela 1. Valores médios dos coeficientes de sensibilidade das variáveis envolvidas no cálculo do fluxo de calor sensível so bre a cultura da cana-de-açúcar

\begin{tabular}{lccccc}
\hline \multirow{2}{*}{ Variáveis } & \multicolumn{4}{c}{ Coeficiente de sensibilidade } \\
\cline { 2 - 6 } & \multicolumn{4}{c}{ Intervalo de medição } & min \\
\cline { 2 - 6 } & $\mathbf{1 5}$ & $\mathbf{3 0}$ & $\mathbf{6 0}$ & $\mathbf{1 2 0}$ \\
Fluxo de calor no solo (Sg) & 0,013 & 0,010 & 0,015 & 0,015 \\
Saldo de radiação (Sr) & 0,988 & 0,853 & 0,993 & 0,993 \\
Temperatura do bulbo seco (St) & 0,158 & 0,158 & 0,158 & 0,148 \\
Temperatura do bulbo úmido (Se) & 0,158 & 0,158 & 0,158 & 0,148 \\
\hline
\end{tabular}

Esta tabela, que sumariza as discussões anteriores em termos das médias dos períodos indica, combinada com a citação anterior de Liqiao et al. (2008), que o saldo de radiação exerce o maior efeito no cálculo da evapotranspiração da cana-de-açúcar dentre as variáveis envolvidas no cálculo do balanço de energia. Por outro lado, o fluxo de calor no solo oferece a menor contribuição sendo que a temperatura do bulbo seco e a temperatura do bulbo úmido desempenham efeitos semelhantes, rigorosamente na mesma magnitude em valor absoluto.

Observa-se ainda que $\mathrm{Sg}$ e $\mathrm{Sr}$ tendem a oferecer uma contribuição maior no cálculo de LE com o aumento do intervalo de medição; inversamente, esta contribuição diminui para as temperaturas dos bulbos seco e úmido. Chen \& Chen (2003) verificaram que a sensibilidade das variáveis aumenta com o aumento do tempo. A constatação no presente estudo, de que o saldo de radiação é a variável mais sensitiva no cômputo do fluxo de calor sensível, está relacionada com a variabilidade da cobertura de nuvens.

A Tabelas 2 apresenta o valor absoluto da média dos erros relativos e o desvio padrão para cada variável envolvida no cálculo do balanço de energia, para o dia 19 de março de 2009. Constata-se um aumento do valor médio do erro relativo com o aumento do intervalo de medição para todas as variáveis envolvidas nessa equação. Apesar das médias dos erros relativos serem pequenas e crescentes, seus desvios padrão

Tabela 2. Valores absolutos da média do erro relativo, em percentagem (\%) e desvio padrão da temperatura do bulbo seco $T_{\text {seco, }}$ fluxo de calor no solo (G), temperatura do bulbo úmido $T_{\text {úmido }}$ e do saldo de radiação $\left(R_{n}\right)$ para 0 dia 19 de março de 2009

\begin{tabular}{|c|c|c|c|c|}
\hline \multirow{4}{*}{$\begin{array}{l}\text { Tempo }(\min ) \\
\text { Média }\left({ }^{\circ} \mathrm{C}\right) \\
\text { Desvio padrão }\left({ }^{\circ} \mathrm{C}\right)\end{array}$} & \multicolumn{4}{|c|}{$\mathrm{T}_{\text {seco }}$} \\
\hline & 15 & 30 & 60 & 120 \\
\hline & 0,002 & 0,014 & 0,012 & 0,085 \\
\hline & 7,600 & 7,480 & 7,350 & 7,120 \\
\hline & \multicolumn{4}{|c|}{ G } \\
\hline Média $\left(\mathrm{Wm}^{-2}\right)$ & 0,055 & 3,080 & 2,940 & 8,189 \\
\hline \multirow[t]{2}{*}{ Desvio padrão $\left(\mathrm{Wm}^{-2}\right)$} & 115,1 & 113,6 & 109,9 & 109,4 \\
\hline & \multicolumn{4}{|c|}{$T_{\text {úmido }}$} \\
\hline Média $\left({ }^{\circ} \mathrm{C}\right)$ & 0,022 & 0,047 & 0,046 & 0,087 \\
\hline \multirow[t]{2}{*}{ Desvio padrão $\left({ }^{\circ} \mathrm{C}\right)$} & 4,370 & 4,330 & 4,240 & 4,030 \\
\hline & \multicolumn{4}{|c|}{$\mathrm{Rn}$} \\
\hline Média $\left(\mathrm{Wm}^{-2}\right)$ & 0,008 & 2,24 & 2,14 & 5,88 \\
\hline Desvio padrão $\left(\mathrm{Wm}^{-2}\right)$ & 58,58 & 57,02 & 51,57 & 50,64 \\
\hline
\end{tabular}


no período analisado praticamente não variaram dentro dos intervalos de medição. Por exemplo, para esse dia a média do erro relativo da $\mathrm{T}_{\text {seco }}$ variou de 0,002 a 0,085 dentro dos intervalos analisados; já a média do erro de $\mathrm{G}$ variou de 0,055 a 8,189; a temperatura do bulbo úmido variou de 0,022 a 0,087 e, finalmente, a média do erro relativo de $\mathrm{R}_{\mathrm{n}}$ variou de 0,008 a 5,88. Os erros relativos das temperaturas dos bulbos seco e úmido são muito pequenos quando comparados com aqueles de $R_{n}$ e G. As maiores médias dos erros relativos ocorrem no intervalo de 120 min, justamente no maior intervalo de medição de todas as variáveis da equação do balanço de energia.

Apresenta-se, na Figura 5, a distribuição dos erros absolutos com os desvios padrão para cada intervalo de medição das temperaturas dos bulbos seco e úmido, fluxo de calor no solo e saldo de radiação na cultura da cana-de-açúcar, no dia 1 de setembro de 2009. Observam-se pequenas oscilações das médias e desvios padrão do erro absoluto da temperatura do bulbo seco durante os intervalos de medição, variando entre $-017 \pm 0,29^{\circ} \mathrm{C}$, no intervalo de $15 \mathrm{~min}$ a $-0,67 \pm 0,29$ ${ }^{\circ} \mathrm{C}$, no intervalo de $120 \mathrm{~min}$ (Figura 5A). Por outro lado, as médias e o desvio padrão do erro absoluto da temperatura do bulbo úmido apresentam comportamento semelhante ao da temperatura do bulbo seco ao longo dos intervalos de medição, muito embora com valores um pouco menores (Figura 5B). A média variou entre $0,03{ }^{\circ} \mathrm{C}$, no intervalo de medição de 30 minutos a $-2,75^{\circ} \mathrm{C}$, no intervalo de $120 \mathrm{~min}$; enquanto o desvio padrão variou do máximo de $0,41^{\circ} \mathrm{C}$, no intervalo de $30 \mathrm{~min}$, a $0,36^{\circ} \mathrm{C}$, no intervalo de $120 \mathrm{~min}$ em face da alta variabilidade da temperatura do ar durante o período seco na região decorrente do efeito da irrigação do canavial. Resultados semelhantes obtiveram Muleta \& Nicklow (2004) e Oyarzun et al. (2006), quando utilizaram a técnica de análise de sensibilidade aplicada à equação de Penman-Monteith.

Os valores das médias e desvios padrão dos erros absolutos do fluxo de calor no solo nos intervalos de medição analisados neste estudo são apresentados na Figura 5C. No intervalo de 15 a 120 min a média variou de $-0,55$ a $-0,35 \mathrm{~W} \mathrm{~m}^{-2}$, enquanto o desvio padrão variou de 7,1 a $6,75 \mathrm{~W} \mathrm{~m}^{-2}$. Constata-se, portanto, que a variação das médias e dos desvios padrão dos erros absolutos do fluxo de calor no solo foi praticamente constante ao longo dos intervalos de medições analisados (Figura 5C). Liebethal et al. (2005) aplicaram a análise de sensibilidade para dois modelos de cálculo do fluxo de calor no solo e verificaram que os sensores instalados em profundidades mais rasas influenciam na qualidade nas medições do que em maiores profundidades.

A distribuição das médias e o desvio padrão dos erros absolutos do saldo de radiação para o dia 1 de setembro são apresentados na Figura 5D. As médias e os desvios padrão para essa variável foram de 17,37 $\pm 119,29 \mathrm{~W} \mathrm{~m}^{-2},-4,96 \pm 127,61$ $\mathrm{W} \mathrm{m}^{-2}, 4,01 \pm 114,65 \mathrm{~W} \mathrm{~m}^{-2} \mathrm{e} 1,96 \pm 11,80 \mathrm{~W} \mathrm{~m}^{-2}$ para os intervalos de medição de 15, 30, 60 e $120 \mathrm{~min}$, respectivamente. Portanto, a ordem de grandeza dos erros absolutos do saldo de radiação é significativamente maior do que para o fluxo de calor solo e, também, o desvio padrão médio nos intervalos de medição do fluxo de calor no solo representa apenas 5\% daquele do saldo de radiação. Este resultado está coerente com as constatações de Azevedo et al. (2007) em que, em média, o fluxo de calor no
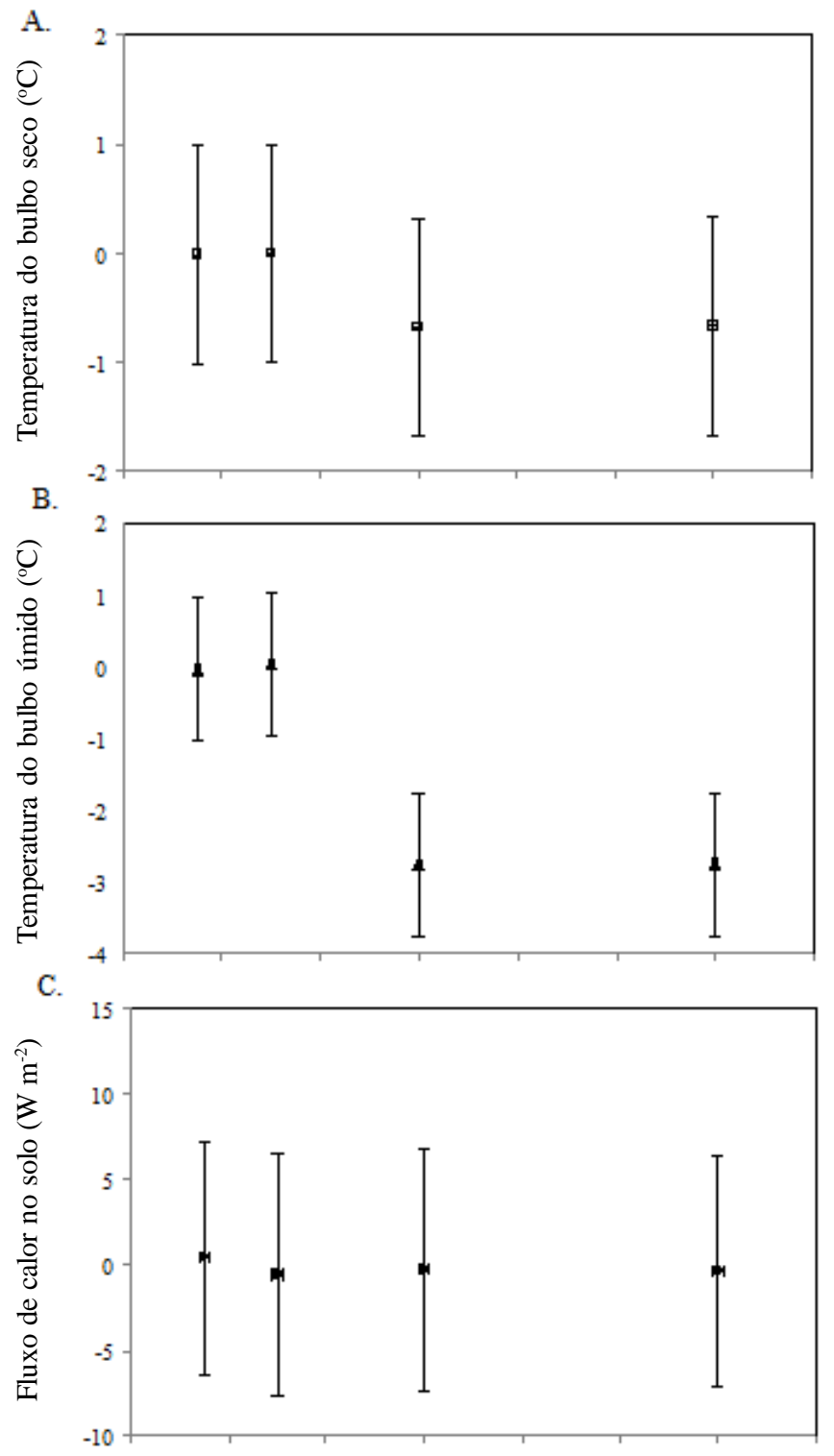

D.

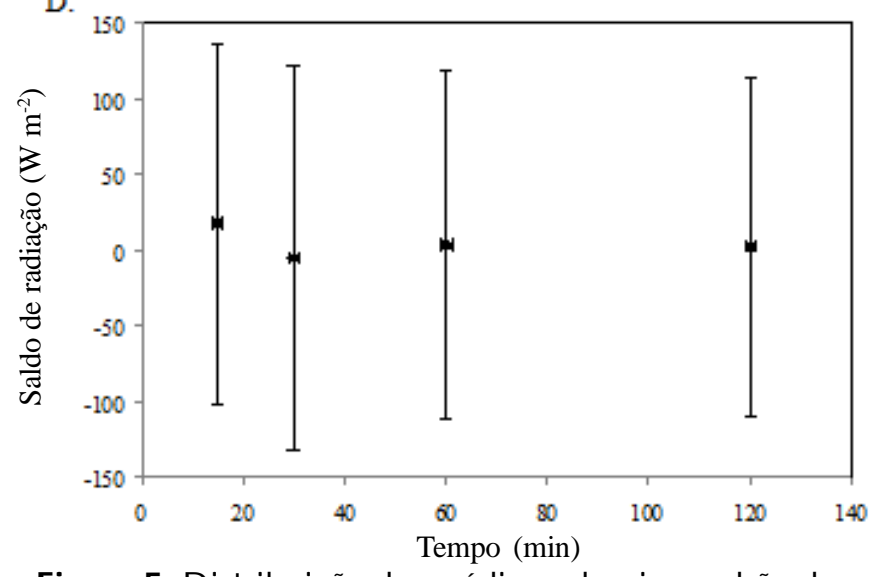

Figura 5. Distribuição das médias e desvios padrão dos erros absolutos para cada intervalo de medição da temperatura do bulbo seco (A), temperatura do bulbo úmido (B), fluxo de calor no solo (C) e saldo de radiação (D), no dia 1 de setembro de 2009 (D)

solo, fluxo de calor sensível e fluxo de calor latente representam, respectivamente, 5,15 e $80 \%$ do saldo de radiação em culturas 
irrigadas. A evapotranspiração estimada pelo balanço de energia apresenta valores bastante satisfatórios, desde que se tenham os devidos cuidados na instalação dos equipamentos para evitar a circulação de calor entre a área de estudo com a superfície seca adjacente (Kang et al., 2008).

\section{CONClusÕES}

1. O saldo de radiação é a variável mais sensitiva no cálculo do balanço de energia, enquanto o fluxo de calor no solo oferece a menor contribuição. Os erros absolutos das variáveis envolvidas no cálculo do balanço de energia aumentam levemente em função do aumento do intervalo de medição.

2. Os erros relativos de todas as variáveis envolvidas do cálculo do balanço de energia baseado na razão de Bowen, aumentam significativamente com o aumento do intervalo de amostragem.

3. A variável mais sensível na determinação da evapotranspiração de referência pelo método de PenmanMonteith é o saldo de radiação, seguido da umidade relativa, velocidade do vento e da temperatura do ar.

\section{LITERATURA CITADA}

Allen, R.G.; Pereira, L.S.; Raes, D.; Smith, M. Crop Evapotranspiration: Guidelines for computing crop water requirements. Rome: FAO, 1998. 297p. Irrigation and Drainage Paper 56.

Azevedo, P. V. de; Silva, B. B. da; Silva, V. de P. R. da. Water requirements of irrigated mango orchard in northeast Brazil. Agricultural Water Management, v.58, p.241-254, 2003.

Azevedo, P. V. de; Sousa, C. B.; Silva, B. B. da; Silva, V. de P. R. da. Water requirements of pineapple crop grown in a tropical environment, Brazil. Agricultural Water Management, v.88, p.201-208, 2007.

Beven, K. A sensitivity analysis of the Penman-Monteith actual evapotranspiration estimates. Journal of Hydrology, v.44, p.169-190, 1979.

Borges, C. J. R.; Azevedo, P. V. de; Silva, V. de P. R. da; Campos, J. H. B. C.; Moura, M. S. B.; Soares, J. M.; Silva, B. B. da. Influência do calor armazenado no sistema solo-planta no balanço de energia em pomar de mangueiras. Revista Brasileira de Engenharia Agrícola e Ambiental, v.12, p.393-399, 2008.

Chen, X.; Chen, X. Sensitivity analysis and determination of streambed leakance and aquifer hydraulic properties. Journal of Hydrology, v.384, p.270-284, 2003.

Gong, L.; Xu, C.; Chen, D.; Halldin, S. Chen, Y. D. Sensitivity of the Penman-Monteith reference evapotranspiration to key climatic variables in the Changjiang (Yangtze River) basin. Journal of Hydrology, v.329, p.3-4, 2006.

Hupet, F.; Vanclooster, M. Effect of the sampling frequency of meteorological variables on the estimation of the reference evapotranspiration. Journal of Hydrology, v.243, p.192-204, 2001.
Hurst, C. A.; Thorburn, P. J.; Lockington, D.; Bristow, K. L. Sugarcane water use from shallow water tables: implications for improving irrigation water use efficiency. Agricultural Water Managenment, v.65, p.1-19, 2004.

Liebethal, C.; Foken, T,; Huwe, B. Sensitivity analysis for two ground heat flux calculation approaches. Agricultural and Forest Meteorology, v.132, p.253-22. 2005.

Liqiao, L.; Bin, L.; Jiuyi, L.; Lijuan, L.; Li, Z. Sensitivity of the Penman-Monteih reference crop avapotranspiration in Taover river basin of northeastern China. Chinese Geographical Science, v.18, p.340-347, 2008.

Kang, S.; Zhang. B.; Li, F.; Zhang, L. Comparasion of three evapotranspiration models to Bowen ratio-energy balance method for a vineyard in an arid desert region of northwest China. Agricultural Journal, v. 148, p.1629-1640, 2008.

Kannan, N.; White, S. M.; Worrall, F.; Whelan, M. J. Sensitivity analysis and identification of the best evapotranspiration and runoff options for hydrological modeling in SWAT-200. Journal of Hydrology, v.332, p.456-466, 2007.

McCuen, R. H. A sensitivity and error analysis of procedures used for estimating evaporation. Water Resource Bulletin, v.10, p.486-498, 1974.

Muleta, M. K.; Nicklow, J. W. Sensitivity and uncertain analysis coupled whit automatic calibration for a distributed watershed model. Journal of Hydrology, v.306, p.127-145, 2004.

Oyarzun, R.; Arumí, J.; Salgado, L.; Mariño, M. Sensitivity analysis and field testing of the RISK-N model in central Valley of Chile. Agricultural Water Management, v.87, p.251-260, 2006.

Rana, G.; Katerji, N. A measurement based sensitivity analysis of the Penman-Monteith actual evapotranspiration model for crops of different height and in contrasting water status. Theoretical and Applied Climatology, v.60, p.141-149, 1998.

Silva, V. de P. R. da. On climate variability in Northeast of Brazil. Journal of Arid Environments, v.58, p.575-596, 2004.

Silva, V. de P. R. da; Azevedo, P. V. de; Silva, B. B. da.Surface energy fluxes and evapotranspiration of mango orchard grown in a semiarid envioronment. Agronomy Journal, v.99, p.1391-1396, 2007.

Silva, V. de P. R. da; Campos, J. H. B. C.; Azevedo, P. V. de. Water-use efficiency and evapotranspiration of mango orchard grown in northeastern region of Brazil. Scientia Horticulturae, v.102, p.467-472, 2009.

Sousa, I. F.; Silva, V. de P. R. da; Sabino, F. G.; Aguiar Netto, A. O.; Silva, B. K. N.; Azevedo, P. V. de. Evapotranspiração de referência nos perímetros irrigados do Estado de Sergipe. Revista Brasileira de Engenharia Agrícola e Ambiental, v.14, p.633-644, 2010.

Souza, C. B.; Silva, B. B. da; Azevedo, P. V. de; Silva, V. de P. R. da. Fluxos de energia e desenvolvimento da cultura do abacaxizeiro. Revista Brasileira de Engenharia Agrícola e Ambiental, v.12, p.400-407, 2008.

Xu, C.; Gong, L.; Jiang, T.; Chen, D.; Singh, V. P. Analysis of spatial distribution and temporal trend of reference evapotranspiration and pan evaporation in Changjiang (Yangtze River) catchment. Journal of Hydrology, v.329, p.620-629, 2006.

Yeh, H. D.; Huang, Y.C. The use of sensitivity analyses in online aquifer parameter estimation. Journal of Hydrology, v.335, p.406-418, 2006. 